\title{
Abdominal Tuberculosis Mimicking Carcinomatosis
}

\section{Ricardo Pedrini Cruz, PhD, Margarete da Rosa Duarte, MD, Gustavo Peretti Rodini, MSc, Anne Rosso Bianchi MD, Marcella Pase Casasola MD, Paula Bibiana Muller Nunes}

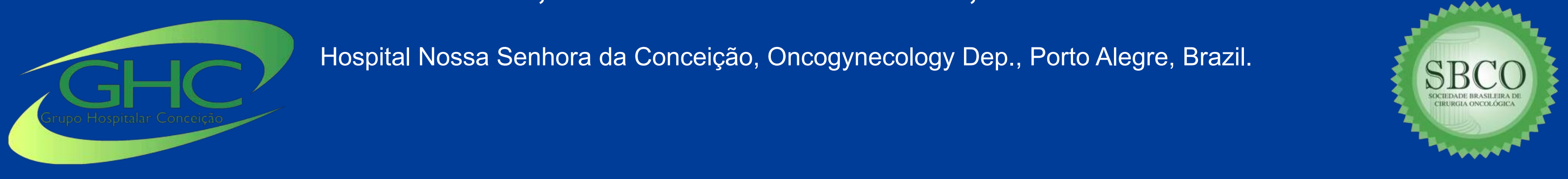

\section{Objectives}

To report an abdominal tuberculosis simulating ovarian cancer. Methods

The information has been obtained through review of medical records and review of the literature.

\section{Case Report}

A 17-year-old female patient was admitted due to fever, abdominal pain, ascites, and previous laparoscopy that evidenced carcinomatosis and ovary lesions. Serum CA-125 was 1529.8 $\mathrm{U} / \mathrm{ml}$ and lactate dehydrogenase (LDH) was 2353

$\mathrm{U} / \mathrm{ml}$. Abdominal magnetic resonance (MR) evidenced lesions with expansive aspect in adnexal regions, suggesting a neoplastic process. Thorax CT was suggestive of active granulomatous infectious process. An uncle died a year ago by tuberculosis and other was being treated for tuberculosis pulmonary infection. HIV serology was negative. Previous laparoscopy material was reexamined, showing active chronic salpingitis with necrotic granulomas, and absence of acid-fast bacilli. A laparoscopy with peritoneal biopsies was performed. Pathology analysis evidenced acid-fast bacilli, compatible with mycobacteriosis. Late cultural examination was compatible with Mycobacterium tuberculosis. She was treated with rifampin, isoniazid, pyrazinamide and ethambutol for two months, followed by four months maintenance treatment with rifampin and isoniazid. Five months after treatment end, the patient was asymptomatic.

\section{Discussion}

Extrapulmonary forms are rarely reported in the pelvis, and most reports are located in the lymph nodes, bones and peritoneum [1]. Risk factors for the development of abdominal TB include HIV infection, cirrhosis, malnutrition, diabetes mellitus, underlying malignancy, immunosuppressive therapy and peritoneal dialysis $[1,2]$. None of these risk factors was present in our patient. In general, clinical manifestations of abdominal TB depend on the form of the disease and may include fever, weight loss, abdominal pain, abdominal distension, ascites, hepatomegaly, diarrhea, and abdominal mass [3]. Patients frequently have family history of TB, which can contribute to the diagnosis. Because abdominal TB has an insidious evolution with nonspecific signs, radiological investigation (preferably by CT or RM) is advised when the disease is suspected, especially in TB endemic areas. Unfortunately, imaging findings can be misdiagnosed as advanced ovarian malignancy or pelvic inflammatory disease [4], being the peritoneal biopsy with pathological examination the only way to discard malignant disease. Patients with abdominal TB should be treated with antituberculosis therapy. The therapeutic response is evidenced by symptoms improvement and decrease in levels of serum CA-125 after two months of treatment [5].

\section{Conclusion}

Tuberculosis should be considered as a differential diagnosis of ovarian cancer, especially in endemic areas

\section{References}

1- Lotfian F, Bolursaz MR, Khalilzadeh S, Baghaie N, Hassanzad M, Velayati A. Features of adolescents tuberculosis at A referral TB's hospital in Tehran, Iran. Mediterr J Hematol Infect Dis. 2016;8: 2016005.

2- Chow KM, Chow VCY, Hung LCT, Wong SM, Szeto CC. Tuberculous peritonitis-associated mortality is high among patients waiting for the results of mycobacterial cultures of ascitic fluid samples. Clin Infect Dis. 2002;35: 409-413.

3- Gostout CJ, Viggiano TR, Balm RK. Acute gastrointestinal bleeding from portal hypertensive gastropathy: prevalence and clinical features. Am J Gastroenterol. 1993;88: 2030-2033. 4- Sah SK, Shi X, Du S, Li X, Li CH, Shah S, et al. CT findings and analysis for misdiagnosis of female pelvic tuberculosis Radiology of Infectious Diseases. 2017;4: 19-25.

5- Bilgin T, Karabay A, Dolar E, Develioglu OH. Peritoneal tuberculosis with pelvic abdominal mass, ascites and elevated CA 125 mimicking advanced ovarian carcinoma: A series of 10 cases. Int J Gynecol Cancer. 2001;11(4):290-4.

Contact

Ricardo Pedrini Cruz - Department of Oncogynecology: Hospital Nossa Senhora da Conceição. Av. Francisco Trein, 596, Porto Alegre, RS, Brazil, 91350-200 Email: rpcruz2004@gmail.com 